Article

\title{
An Improved Mixed Integer Linear Programming Approach Based on Symmetry Diminishing for Unit Commitment of Hybrid Power System
}

\author{
Bo Fu ${ }^{1}$, Chenxi Ouyang ${ }^{1}$, Chaoshun $\mathrm{Li}^{2, *}$, Jinwen Wang ${ }^{2}$ and Eid Gul ${ }^{3}$ \\ 1 School of Electrical and Electronic Engineering, Hubei University of Technology, Wuhan 430068, China; \\ fubofanxx@yahoo.com.cn (B.F.); koalaoycx@gmail.com (C.O.) \\ 2 School of Hydropower and Information Engineering, Huazhong University of Science and Technology, \\ Wuhan 430074, China; jinwen.wang@hust.edu.cn \\ 3 China-EU Institute for Clean and Renewable Energy, Huazhong University of Science and Technology, \\ Wuhan 430074, China; i201721109@hust.edu.cn \\ * Correspondence: csli@hust.edu.cn; Tel.: +86-158-7180-0142
}

Received: 30 January 2019; Accepted: 26 February 2019; Published: 3 March 2019

\begin{abstract}
In this paper, the mixed integer linear programming (MILP) for solving unit commitment (UC) problems in a hybrid power system containing thermal, hydro, and wind power have been studied. To promote its efficiency, an improved MILP approach has been proposed, while the symmetric problem in MILP formulas has been solved by reforming hierarchical constraints. Experiments on different scales have been conducted to demonstrate the effectiveness of the proposed approach. The results indicate a dramatic efficiency promotion compared to other popular MILP approaches in large scale power systems. Additionally, the proposed approach has been applied in UC problems of the hybrid power system. Two indexes, fluctuation degree and output degree, have been proposed to investigate the performance of renewable energy sources (RES). Several experiments are also implemented and the results show that the integration of pumped hydroelectric energy storage (PHES) can decrease the output of thermal units, as well as balance wind power fluctuation according to the load demand.
\end{abstract}

Keywords: unit commitment; mixed integer linear programming (MILP); symmetry diminishing; improved hierarchical constraints; PHES; renewable energies; RES

\section{Introduction}

The unit commitment (UC) optimization is a large-scale, non-convex, and mixed-integer linear optimization problem that is hard to solve. As a kind of branch-and-cut based algorithm, MILP is one of the effective methods for solving the UC problems in large-scale systems [1]. Numerous studies have devoted to either improve the MILP algorithms or enlarge the application of MILP algorithms in various applications. Among these, the symmetry phenomena in UC problems and MILP formulas have attracted great attention from researches. In the UC problem, symmetric situation occurs frequently. Especially in large scale system, scheduling identical generators originates an algorithmic issue known as the symmetric problem. The symmetric problem emerges in the MILP formula if the variables can be substituted without changing the structure of mixed integer linear problem. Plenty of studies have proved that the symmetry may have great influence on the performance of MILP, while this problem will produce redundant calculation for exploring equivalent search regions unnecessarily [2].

The methods for solving a symmetric problem can be categorized into two main methodologies: Reformulation methods and removal methods [1]. In reformulation methods, isomorphism pruning [3] 
and orbital branching [4] are often used to reduce symmetry. However, these methods require rewriting formulations that are not easy tasks and this could lead to a risk in which the dimension of the solving problem will increase. In removal methods, dynamically and statically constraints are used for symmetry diminishing. The dynamically one reduces symmetry during the tree searching process and an additional software called off-the-shelf need to be implemented. The other one is much more simple and exploits symmetry only by adding hierarchies in the selection process and no special software is needed. Therefore, this study static removal strategy is taken to set priorities to the generators status variables.

Actually, adding hierarchical constraints into MILP can be supported by many related efforts and articles. Yokoyama et al. [5] utilized hierarchical relationships between design and operation variables to search the K-best solutions. The results demonstrated that their work is superior to the conventional method with the solution optimality and computation efficiency are improved a lot. Lima et al. [6] made a comparison between the case with and without symmetry breaking constraints. The results of their study state that symmetry breaking constraints performance well in UC MILP models without having a significant impact on the size of the model. Alemany et al. [1] presented a way to reduce the computational burden of the Branch and Cut algorithm without changing the structure of the problem. In these works, the methodology proposed by Alemany et al. [1] lead to a considerable reduction of the search for the branch-and-cut enumeration tree without complex constraints, which is much more superior to other works. Unfortunately, with the system scale increasing, the advantage of their approach is becoming weak. Therefore, this paper presents an improved symmetry breaking approach, which is derived from Reference [1] to diminish symmetry in an efficient way. The UC MILP model comes from $[7,8]$ is taken in this paper without considering ramping up and down constraints as these constraints may prevent the utilization of symmetry breaking constraints [6].

In addition, renewable energy sources (RES), regarded as clean and economical energy sources, are likely to be connected to power systems $[9,10]$ for distributed utilization; the study of UC for power systems containing RES has become a popular topic in research and applications [11]. Thus, in this paper, a hybrid power system is implemented with thermal, hydro, and wind to investigate the UC problems of hybrid systems. The hybrid system is shown in Figure 1.

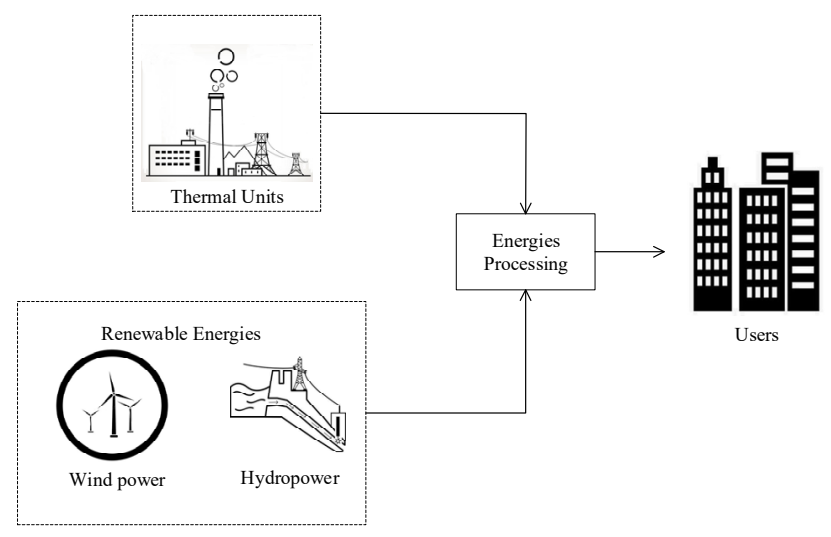

Figure 1. The hybrid power system.

In a hybrid power system, the energy storage system (ESS) is one of the most critical components. The ESS is an indispensable part of a hybrid power system, because the use of an ESS can not only improve power quality, but also ensure the stable and reliable operation of the whole system [12,13]. Recently, pumped hydroelectric energy storage (PHES) is demonstrated to be one of the most effective ESSs for the hybrid system, showing striking advantages on capacity, cost and sufficiency [11,14]. Therefore, this study uses PHES as an ESS to decrease load fluctuation, which was caused by RES integration. Furthermore, in order to discuss the performance of PHES in detail, two indexes are presented: Firstly, an index called fluctuation degree is introduced in this paper to assess load 
fluctuation with and without PHES integration. What is more, considering the performance of PHES is closely related to two factors: System scale and capacity of storage; a new index called output degree is proposed to evaluate the influence of these factors on PHES output.

From the above discussion, we were motivated to present an improved MILP model for UC of hybrid power systems integrating RES and investigate the impact of different factors on PHES in detail. The main contributions of this article are: (1) An improved MILP model based on hierarchical constraints has been proposed and applied in different scales of UC problems; (2) an index called fluctuation degree is presented to evaluate the impact of RES integration; and (3) an index called output degree has been proposed to investigate PHES output. The framework of this paper is presented in Figure 2.

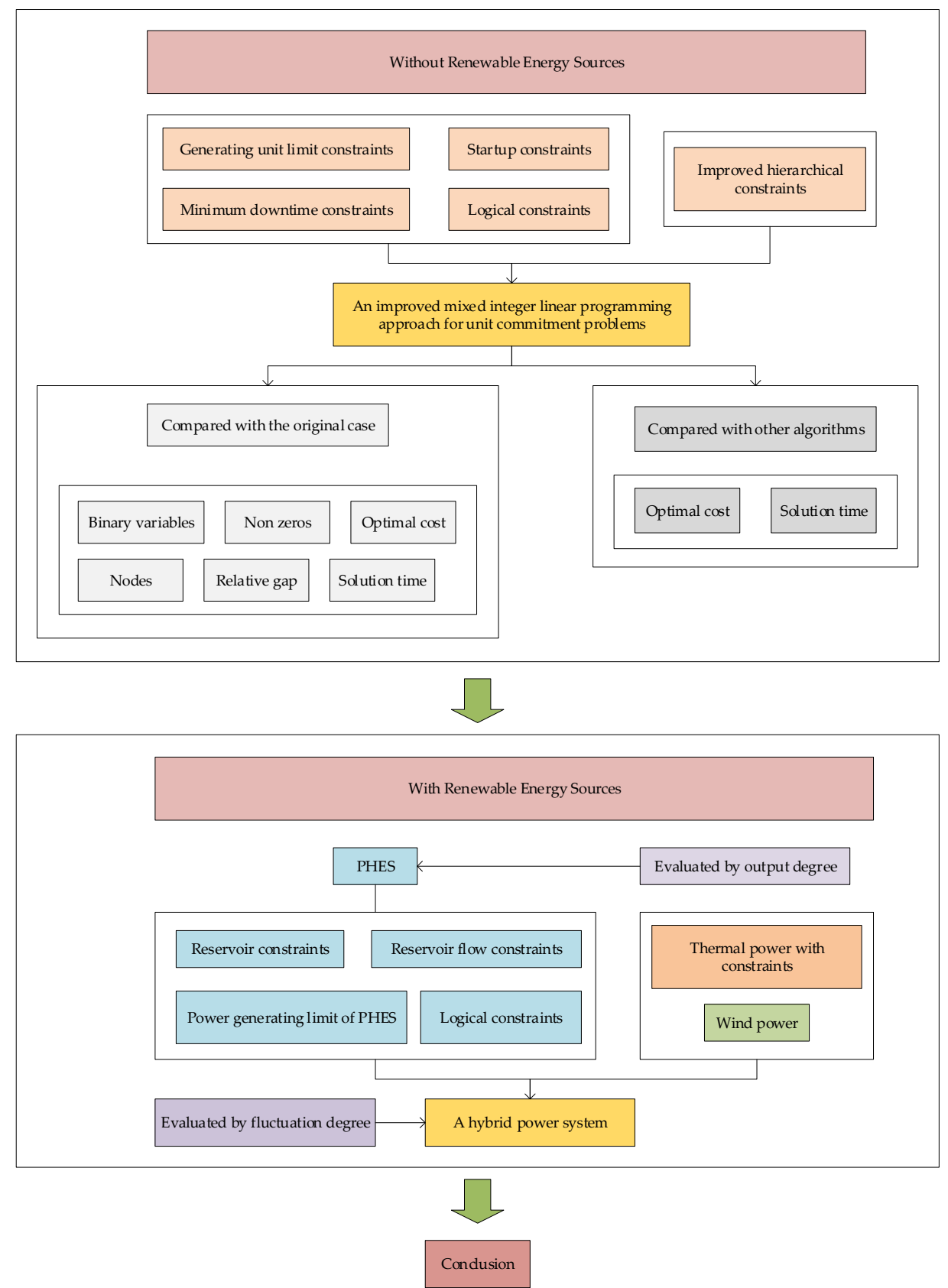

Figure 2. The framework of the improved approach.

The rest part of the paper is organized as follows: Section 2 gives the MILP formulations for UC problems of hybrid systems are introduced. Section 3 describes the proposed MILP approach 
with symmetry breaking constraints. Section 4 validates the proposed MILP model in comparative experiments, while the impact of RES in different system scales and the impact of two factors of PHES are evaluated. The conclusions are drawn in Section 5.

\section{Formulations for UC Problem}

The hybrid system, which consists of thermal units, PHES and wind power is studied in this paper. To minimize operational cost is commonly the objective target. The unit commitment problem can be formulated as [7]:

$$
\min f=\sum_{t=1}^{T} \sum_{i=1}^{G e n}\left[C p_{i, t}+C u_{i, t}\right]
$$

where:

$$
\begin{aligned}
C p_{i, t} & =a_{i} \text { onoff } f_{i, t}+b_{i} p_{i}+c_{i} p_{i}^{2} \\
C u_{i, t} & =z_{i, k, 1} H S T_{i}+z_{i, k, 2} C S T_{i}
\end{aligned}
$$

Subject to:

$$
\begin{gathered}
\text { Load }_{t}=\sum_{t=1}^{T} \sum_{i=1}^{G e n}\left(p_{i, t}+P h_{t}+P w_{t}\right) \\
\sum_{t=1}^{T} \sum_{i=1}^{G e n} \text { onoff }_{i, t} P_{i \max }+P h_{t}+P w_{t}-\text { Load }_{t} \geq \text { reserve }_{t} \\
C u_{i, t}, p_{i, t}, P h_{t}, P w_{t} \in \prod_{i, t}
\end{gathered}
$$

where $\prod_{i, t}$ represents the set of feasible production quantities for generator $j$ in time period $i$. The function Load $_{i}$ gives the demand at time $t$, while reserve $t$ gives the spinning reserve requirement. The function $C p_{i, t}$ that describes the cost of generation is generally assumed a quadratic function. Typically, it is modeled as a piecewise linear function. Perspective cuts, which is known as a linear approximation, are given in References $[15,16]$ in detail. $a, b$ and $c$ are the parameters of generating. Function $C u_{i, t}$ is the cost of startup. $p_{i, t}, P h_{t}$ and $P w_{t}$ give the thermal, hydro and wind power output in the time period, respectively. In addition, onof $f_{i, t}$ is the generators status variable. $z_{i, t, 1}$ and $z_{i, t, 2}$ are the status variables of hot and cold startup, respectively. This paper is focused on the set of constraints in Equation (6).

\subsection{Constraints for Thermal Units}

The thermal constraints are including generation limit constraint, cold/hot startup constraint, minimum down time constraint, and logical constraint [7]. The first one limits the thermal unit outputs. The next one chooses the cold or hot startup mode. The third one could control the unit's downtime as generators are not allowed to re-start immediately and the last constraint can ensure the logical of variables in UC problems. These constraints can be one or more in the following (7)-(10).

(1) Generating Unit Limit Constraints

$$
P_{\text {imin }} \cdot \text { onoff }_{i, t} \leq p_{i, t} \leq P_{i \max } \cdot \text { onoff }_{i, t}
$$

(2) Startup Constraints

$$
\left\{\begin{array}{l}
z_{i, t, 1}+z_{i, t, 2} \leq y_{i, t, 1} \\
z_{i, t, 2} \geq y_{i, t, 1}-\sum_{k=D T+1}^{D T+T o l d+1} \text { onoff } f_{i, t-k}
\end{array}\right.
$$


(3) Minimum Downtime Constraints

$$
\sum_{k=\max (t-D T+1,1)}^{t} y_{i, k, 2} \leq 1-\text { onoff }_{i, t}, t \in\left[L_{i}+1, T\right]
$$

$y_{i, t, 1}$ and $y_{i, t, 2}$ represent the status of startup and shutdown, respectively. DT is the minimum down time of generators and Tcold is the minimum cold time. $L_{i}$ is the continuous down time of unit $i$ at $t_{0} . L_{i}=\max \left\{0, \min \left[T,\left(1-\right.\right.\right.$ onoff $\left.\left.\left._{i, 0}\right)\left(D T_{i}+T_{i, 0}\right)\right]\right\}$, in this equation, onof $f_{i, 0}$ is the original state of unit $i ; T_{i, 0}$ is the continuous up (positive value)/down (negative value) time before $t_{0}$.

(4) Logical Constraint

$$
\text { onoff } f_{i, t}-\text { onoff }_{i, t-1}=y_{i, t, 1}-y_{i, t, 2}
$$

\subsection{Constraints for PHES}

PHES undertakes the task of regulating the peak value and filling valley to save energy in power systems [11,17]. In this part, up/down reservoir constraints, up/down reservoir flow constraints, logical constraints, and power generation of PHES constraints are been taken from Reference [18]. The first one is use to constrain the capacity of up and down reservoirs. The second one presents the flow limitation of up and down reservoirs. The formulation of the third one is the same as Equation (10) to ensure variables' logical and the power generation of PHES is controlled by the last one.

(1) Reservoir Constraints

$$
\left\{\begin{array}{l}
V_{\min }^{u} \leq V_{t}^{u} \leq V_{\max }^{u} \\
V_{\min }^{d} \leq V_{t}^{d} \leq V_{\max }^{d}
\end{array}\right.
$$

(2) Reservoir Flow Constraints

When $P h_{t}>0$, PHES is working on generating mode. As the capacity of up reservoir decreasing, the capacity of down reservoir will increase. $\Delta t$, which represents a time interval, equals $1 \mathrm{~h}$ in this paper.

$$
\left\{\begin{array}{l}
-u_{2 t} \cdot M<=V_{t+1}^{u}-V_{t}^{u}+\Delta t P h_{t} / \eta_{1}<=u_{2 t} \cdot M \\
-u_{2 t} \cdot M<=V_{t+1}^{d}-V_{t}^{d}-\Delta t P h_{t} / \eta_{1}<=u_{2 t} \cdot M
\end{array}\right.
$$

On the contrary, when $P h_{t}<0$, PHES is working on pumping. The rising capacity of up reservoir comes the decreasing capacity of the down reservoir.

$$
\left\{\begin{array}{l}
-u_{1 t} \cdot M<=V_{t+1}^{u}-V_{t}^{u}+\Delta t P h_{t} \cdot \eta_{2}<=u_{1 t} \cdot M \\
-u_{1 t} \cdot M<=V_{t+1}^{d}-V_{t}^{d}-\Delta t P h_{t} \cdot \eta_{2}<=u_{1 t} \cdot M
\end{array}\right.
$$

In addition, $\eta_{1}$ and $\eta_{2}$ are fixed constants, which describe the efficiency of generating and pumping, respectively, while ignoring the change of water-head in PHES.

(3) Logical Constraints

$$
u_{1 t}+u_{2 t}=1
$$

(4) Power Generating limits of PHES

$$
P h_{t \min } \leq P h_{t} \leq P h_{t \max }
$$

Where

$$
\left\{\begin{array}{l}
P h_{t \min }=\max \left[-P P_{\max }-V_{t}^{d} /\left(\Delta t \eta_{2}\right)\right] \\
P h_{t \max }=\min \left[P G_{\max }\left(V_{t}^{u} / \Delta t\right) \cdot \eta_{1}\right]
\end{array}\right.
$$




\subsection{Wind Power Generation}

The outputs of wind turbines are different even though they install in the same place and with the same rated power. Therefore, through a comprehensive literature review, Equation (17), which comes from Reference [19] is adopted in this paper to generate wind power for a given wind speed input.

$$
P w_{t}= \begin{cases}0 & \text { for } v_{t}<v_{i} \text { and } v_{t}>v_{0} \\ p_{r}\left(\frac{v_{t}-v_{i}}{v_{r}-v_{i}}\right) & \text { for } v_{i} \leq v_{t} \leq v_{r} \\ p_{r} & \text { for } v_{r} \leq v_{t} \leq v_{0}\end{cases}
$$

where $p_{r}$ is the rated power. $v_{t}$ is the wind speed (in $\mathrm{m} / \mathrm{sec}$ ), and $v_{i}, v_{0}$ and $v_{r}$ are the cut-in, cut-out and rated wind speeds, respectively.

\section{New Formulation based on System Breaking}

\subsection{Symmetry in Mixed Integer Linear Programming}

The Mixed integer linear programming (MILP) problem can be defined as:

$$
\min \left\{c x+h y: A x+G y \geq b, x \in Z^{n}, y \in \Re^{p}\right\}
$$

where $A$ and $G$ are $m \times n$ matrices, $c$ and $h$ are $n-$ vectors, and $b$ is $m$ - vector. $x, y$ are variables, where $x$ is the integer variable and $y$ is the continuous variable.

The set of all feasible solutions is $Q$. From $Q$, it is obvious that the MILP problem that describes in Equation (18) may induce multiple equivalent solutions, each of them consisting a symmetry group $G$. Further details can be found in Reference [2]. Mathematically, the symmetry group $G$ of the problem proposed in Equation (19) can be regarded as the set of all permutations $\pi$ of the $n$ variables mapping $Q$ on itself and mapping each feasible solution on a feasible solution within the same objective value:

$$
G=\left\{\pi \in \prod^{n} \mid \forall x \in Q: \pi(x) \in Q \wedge c^{T} x=c^{T} \pi(x)\right\}
$$

If the equivalence of these sub-problems is uncertain, this may lead to solving unnecessary problems which will make easy problem become complex because of redundant calculations. Therefore, the main challenge of symmetry breaking problem is to discern a subset of a symmetry group in order to relieve the computational burden.

The static symmetry method is effective by constructing hierarchical constraints and adding them to the initial formulations. These methods ensure some of the symmetric solutions breaking, while at least one optimal solution keeping. Generally, the static symmetry can divide into two options: One is to compute a subgroup $G_{L P}$ of $G$; another is to impose hierarchical decisions. However, another option is to utilize the knowledge of the model. In Reference [20], Sherali H.D. and Smith J.C. proposed an alternative to this knowledge-based method. The authors impose hierarchical decision to decrease the symmetry effect on their model.

In UC formulations, the symmetry problems are driven by the identical units' status variables in the system. In a given scheduling pattern with identical units, there are several identical patterns can be found by permuting the state variables among the identical generator. Therefore, this paper proposed hierarchical decisions, which were suggested by Reference [20].

To describe the symmetry breaking theory in UC problem, the example in Reference [1] would be interesting. In this example, a set $Q$ containing 9 feasible solutions can be represented as follows: 


$$
Q=\left\{\begin{aligned}
\{[0,1,0,1]\} & \mapsto f_{1}=186 \\
\{[1,0,1,0]\} & \mapsto f_{2}=186 \\
\{[0,1,1,0]\} & \mapsto f_{3}=196 \\
\{[1,0,0,1]\} & \mapsto f_{4}=196 \\
\{[0,1,1,1]\} & \mapsto f_{5}=204 \\
\{[1,0,1,1]\} & \mapsto f_{6}=204 \\
\{[1,1,0,1]\} & \mapsto f_{7}=204 \\
\{[1,1,1,0]\} & \mapsto f_{8}=204 \\
\{[1,1,1,1]\} & \mapsto f_{9}=212
\end{aligned}\right.
$$

1 and 0 here are the values of onoffi,t they are represented as vectors [onof $f_{1,1}$, onof $f_{2,1}$, onof $f_{1,2}$, onof $f_{2,2}$, and the corresponding objective value is $f_{i}$. It obviously that some permutation of the solutions have the equivalent $f$.

\subsection{Improvements on Hierarchical Constraints}

The author in Reference [1] added the hierarchical constraints to MILP for symmetry diminishing, the inequality is described in the following:

$$
\text { onoff } f_{1, t} \geq \text { onoff } f_{2, t}
$$

After solving UC MILP with Equation (20), the set $Q$ can be reduced into four feasible solutions:

$$
Q=\left\{\begin{aligned}
\{[1,0,1,0]\} & \mapsto f_{1}=186 \\
\{[1,0,1,1]\} & \mapsto f_{2}=204 \\
\{[1,1,1,0]\} & \mapsto f_{3}=204 \\
\{[1,1,1,1]\} & \mapsto f_{4}=212
\end{aligned}\right.
$$

Even though there are two solutions of 204, the set $Q$ decreased a lot that verifies the efficient performance of hierarchical constraints. Equation (20) ensures the order of the binary vectors to avoid redundant calculations.

However, as the scale of power systems increasing, the performance of Equation (20) is not working well with symmetry problems aggrandizing. Therefore, this paper proposed a slight improvement on the Equation (20) without considering hierarchies one by one. The improved hierarchical constraints, which adjust the order of symmetry diminishing, can improve the performance of MILP in the large scale of the systems. The proposed constraints are represented as follows:

$$
\begin{gathered}
\operatorname{onoff}_{1, t} \geq \operatorname{onoff}_{3, t} \geq \ldots \geq \operatorname{onoff}_{2(n-1), t} n \in B_{G e n} \\
\text { onoff }_{2, t} \geq \text { onoff }_{4, t} \geq \ldots \geq \text { onoff }_{2 n, t} n \in B_{G e n}
\end{gathered}
$$

Generally, the improved hierarchical algorithm outlines of this paper can be written in the following steps:

1. Identify the identical generating units from the total group $G$.

2. Form a subset $B_{G e n}$ of $G$.

3. Activate the units from the highest order to meet the load and spinning reserves.

4. Turn on units in lower order if needed.

5. Keep all the lower units deactivate if the higher one is not activated.

6. Solve for economic dispatch in UC. 


\section{Results of Experiments}

To be honestly evaluate the performance of improved constraints, UC problems with different scales are studied in the following experiments. In addition, two indexes have been used to evaluate the performance of RES.

\subsection{Efficiency Test}

In order to test the computational efficiency of the proposed approach, a popular power system with 10 thermal units are selected. Based on the 10-unit system, the cases with 20, 40, 60, 80, and 100-units were established by duplication. Both the basic data of the thermal units and $24 \mathrm{~h}$ load are from Reference [21]. The spinning reserve is set to $10 \%$ load demand in this and the latter experiments.

\subsubsection{Computational Results with Improved Constraints}

The model is implemented in MATLAB using Gurobi as the solver, with default gap set to $0.05 \%$. Each case is solved considering the basic approach (BA) [1] as well as the improved approach (IA) proposed in this paper. Additionally, in order to do a fair comparison between two approaches, different gap values $0.1 \%, 0.5 \%$, and $1 \%$ have been chosen to verify their results. The simulation results are exhibited in Table 1.

Table 1. Comparison of performance between different hierarchical constraints.

\begin{tabular}{cccccc}
\hline \multirow{2}{*}{ Index } & Approach & \multicolumn{5}{c}{ Units } \\
\cline { 3 - 6 } & & $\mathbf{4 0}$ & $\mathbf{6 0}$ & $\mathbf{8 0}$ & $\mathbf{1 0 0}$ \\
\hline \multirow{2}{*}{ B Variables } & BA & 6720 & 10,080 & 13,440 & 16,800 \\
& IA & 6720 & 10,080 & 13,440 & 16,800 \\
Non zeros & BA & 49,136 & 73,944 & 98,752 & 123,560 \\
& IA & 48,656 & 73,464 & 98,272 & 123,080 \\
Optimal Cost & BA & $2,242,974$ & $3,361,385$ & $4,482,150$ & $5,600,467$ \\
& IA & $2,242,596$ & $3,360,419$ & $4,480,326$ & $5,598,415$ \\
Nodes & BA & 19 & 283 & 1320 & 1971 \\
& IA & 1369 & 1402 & 1325 & 356 \\
Relative gap & BA & $1.0 \times 10^{-5}$ & $1.2 \times 10^{-5}$ & $2.0 \times 10^{-5}$ & $5.0 \times 10^{-5}$ \\
Solution time & IA & $2.0 \times 10^{-6}$ & $2.5 \times 10^{-5}$ & $1.8 \times 10^{-5}$ & $4.3 \times 10^{-5}$ \\
(gap: default) & BA & 6.56 & 16 & 40.11 & 126.67 \\
Solution time & IA & 15.58 & 34.88 & 26.45 & 26.66 \\
(gap: 0.1\%) & BA & 6.02 & 11.44 & 19.02 & 22.41 \\
Solution time & IA & 5.73 & 8.58 & 13.05 & 19.13 \\
(gap: 0.5\%) & BA & 2.05 & 4.3 & 9.06 & 13.3 \\
Solution time & IA & 2.08 & 4.02 & 5.72 & 14.92 \\
(gap: 1\%) & BA & 1.3 & 4.06 & 7.8 & 11.06 \\
\hline
\end{tabular}

Table 1 presents that IA can get a better optimal value with less time cost than BA, while the relative gap is narrow in most cases.

Furthermore, these two approaches will be compared in large scale system with default gap set to $1 \%$. Optimal cost and solution time are as the indexes to evaluate the performance of proposed constraints. The simulation results are shown in Table 2.

Table 2 describes that in large scale systems, from the index of optimal cost and solution time, IA performance better in most cases. In the 600,700, and 1000-units cases, the solution times of IA are more than BA. However, if the gap set to $2 \%$, the solution times of IA in these cases are $816.13 \mathrm{~s}$, $873.5 \mathrm{~s}$ and $1222.14 \mathrm{~s}$, respectively, which are less than the solution times of BA (1193.52 s, $1249.78 \mathrm{~s}$ and 1446.11s). Similarly, the optimal costs of IA are more than BA in 200, 600, 700, 800, and 900-units cases. If the gap in these cases is set to $2 \%$, the advantages of IA will be validated. Therefore, Table 3 presents the results of simulation with the gap set to $2 \%$. 
Table 2. Comparison of performance in large scale (gap: 1\%).

\begin{tabular}{ccccc}
\hline \multirow{2}{*}{ Scale } & \multicolumn{2}{c}{ Optimal Cost } & \multicolumn{2}{c}{ Solution Time } \\
\cline { 2 - 5 } & BA & IA & BA & IA \\
\hline 100 & $5,636,172$ & $5,634,435$ & 25 & 9 \\
200 & $11,274,242$ & $11,293,907$ & 118 & 45 \\
300 & $16,925,682$ & $16,922,865$ & 212 & 109 \\
400 & $22,552,186$ & $22,550,605$ & 259 & 250 \\
500 & $28,205,128$ & $28,177,326$ & 575 & 337 \\
600 & $33,794,489$ & $33,856,539$ & 1621 & 683 \\
700 & $39,466,484$ & $39,497,405$ & 674 & 686 \\
800 & $45,063,024$ & $45,167,260$ & 1946 & 867 \\
900 & $50,734,953$ & $50,792,242$ & 2684 & 1416 \\
1000 & $56,347,579$ & $55,916,000$ & 4505 & 5137 \\
\hline
\end{tabular}

Table 3. Comparison of performance in large scale (gap: $2 \%$ ).

\begin{tabular}{ccccc}
\hline \multirow{2}{*}{ Scale } & \multicolumn{2}{c}{ Optimal Cost } & \multicolumn{2}{c}{ Solution Time } \\
\cline { 2 - 5 } & BA & IA & BA & IA \\
\hline 100 & $56,909,362$ & $56,494,104$ & 16.2 & 12 \\
200 & $11,392,386$ & $11,343,173$ & 46.47 & 49.97 \\
300 & $16,923,744$ & $16,864,586$ & 356 & 94.91 \\
400 & $22,668,351$ & $22,613,340$ & 322 & 243.28 \\
500 & $28,289,153$ & $28,352,109$ & 588.72 & 171.39 \\
600 & $33,924,776$ & $33,868,078$ & 619.02 & 516.3 \\
700 & $39,867,856$ & $39,580,050$ & 553.39 & 679.35 \\
800 & $45,420,972$ & $45,246,954$ & 1193.52 & 816.13 \\
900 & $50,942,836$ & $50,873,907$ & 1249.78 & 873.5 \\
1000 & $56,770,346$ & $56,569,896$ & 1446.11 & 1222.14 \\
\hline
\end{tabular}

The results demonstrate that IA uses less time to obtain better optimal cost with an appropriate gap value.

\subsubsection{Comparison with Other MILP Approaches}

In order to validate the performance of the proposed approach, some popular MILP algorithms are adopted for comparison, including EPL (Extended Priority List) [21], DPLR (Adaptive Lagrangian Relaxation) [22], LSLR (Local Search Lagrangian Relaxation) [23], and SHCMIP [24]. The results are presented in Tables 4 and 5, respectively.

Table 4. Comparison of optimal cost between different algorithms (\$).

\begin{tabular}{cccccc}
\hline Scale & EPL [21] & DPLR [22] & LSLR [23] & SHCMIP [24] & IA \\
\hline 10 & 563,977 & 564,049 & 564,970 & 563,978 & 563,938 \\
20 & $1,124,369$ & $1,128,098$ & $1,125,064$ & $1,123,342$ & $1,123,299$ \\
40 & $2,246,508$ & $2,256,195$ & $2,242,968$ & $2,243,079$ & $2,242,595$ \\
60 & $3,366,210$ & $3,384,293$ & $3,361,244$ & $3,361,766$ & $3,360,339$ \\
80 & $4,489,322$ & $4,512,391$ & $4,482,403$ & $4,482,103$ & $4,480,327$ \\
100 & $5,608,440$ & $5,640,488$ & $5,600,457$ & $5,601,954$ & $5,598,290$ \\
\hline
\end{tabular}


Table 5. Comparison of computing time between different algorithms (s).

\begin{tabular}{cccccc}
\hline Scale & EPL [21] & DPLR [22] & LSLR [23] & SHCMIP [24] & IA \\
\hline 10 & 0.72 & 108 & 2.80 & 0.96 & 0.34 \\
20 & 2.97 & 299 & 5.40 & 4.59 & 1.34 \\
40 & 11.9 & 1200 & 13.5 & 7.54 & 16.91 \\
60 & 23.0 & 3199 & 25.8 & 41.4 & 18.20 \\
80 & 44.4 & 8447 & 39.7 & 69.6 & 26.77 \\
100 & 64.5 & 12,437 & 61.9 & 83.7 & 52.33 \\
\hline
\end{tabular}

\subsection{Solutions of UC Problems for Hybrid Power System}

In this subsection, the MILP formulations for UC problems of hybrid power systems containing thermal, hydro and wind power have been evaluated. The PHES data is cited from Reference [18] and the wind data is referred to from Reference [19].

\subsubsection{UC results with Different Hybrid System Compositions}

In this subsection, the improved MILP approach will be applied in UC problems of hybrid power systems with different scales from 100-unit to 1000-unit, with default gap set to $1 \%$. A new index called fluctuation degrees is defined to illustrate load fluctuation with RES integration.

$$
I_{\text {fluct }}=\frac{1}{T} \sum_{t=1}^{T}\left(\text { net_load }_{t}-a v g_{t}\right)^{2}
$$

where net_load is the net load and avg is the mean value of net load. net_load and avg are defined as follows:

$$
\begin{aligned}
& \text { net_load } t= \begin{cases}\text { Load }_{t} & \text { for thermal system } \\
\text { oad }_{t}-P w_{t} & \text { for thermal }- \text { wind system } \\
\text { Load }_{t}-P w_{t}-P h_{t} & \text { for thermal }- \text { wind }- \text { PHES system }\end{cases} \\
& \operatorname{avg}_{t}=\frac{1}{T} \sum_{t=1}^{T} n e t \_l o a d_{t}
\end{aligned}
$$

The key specifications of wind turbine have been exhibited in Table 6, and the optimal cost and the fluctuation index between two situations have been described in Table 7. The results demonstrate that: (1) The operation cost of thermal units steadily decreases as the integration of RES; and (2) the integration of PHES could restrain the load fluctuation efficiently.

Table 6. Key specifications of the wind turbine.

\begin{tabular}{cccc}
\hline Rated Power & Rated Wind Speed & Cut in Wind Speed & Cut off Wind Speed \\
\hline $240 \mathrm{MW}$ & $5 \mathrm{~m} / \mathrm{s}$ & $3.5 \mathrm{~m} / \mathrm{s}$ & $25 \mathrm{~m} / \mathrm{s}$ \\
\hline
\end{tabular}

In conclusion, the combination of PHES in the hybrid system can not only decrease the power output of thermal units, but can also balance the load fluctuation with RES integrating. The fluctuation of the thermal unit output becomes narrow which would be beneficial for reducing operational frequency of startup/shutdown and smoothing power variation, contributing to a decrease in operational cost. 
Table 7. Comparison of the improved MILP of UC problems results in different hybrid systems.

\begin{tabular}{ccccc}
\hline \multirow{2}{*}{ Scale } & \multicolumn{2}{c}{ Cost (\$) } & \multicolumn{2}{c}{$\mathbf{I}_{\text {fluct }}$} \\
\cline { 2 - 5 } & Thermal-Wind & Thermal-Wind-PHES & Thermal-Wind & Thermal-Wind-PHES \\
\hline 100 & $4,991,126$ & $4,932,701$ & $5,869,100$ & $52,365,00$ \\
200 & $10,021,498$ & $9,894,946$ & $23,478,000$ & $20,464,000$ \\
300 & $15,022,335$ & $14,853,533$ & $52,822,344$ & $46,329,000$ \\
400 & $20,049,056$ & $19,800,901$ & $93,906,389$ & $82,330,000$ \\
500 & $25,018,330$ & $24,733,338$ & $146,730,000$ & $128,160,000$ \\
600 & $30,040,031$ & $29,732,068$ & $211,289,375$ & $183,430,000$ \\
700 & $34,915,518$ & $34,632,363$ & $287,588,316$ & $248,640,000$ \\
800 & $39,840,756$ & $39,559,527$ & $375,630,000$ & $328,080,000$ \\
900 & $45,027,102$ & $44,363,237$ & $475,401,094$ & $406,490,000$ \\
1000 & $50,128,293$ & $49,533,136$ & $8,924,857,149$ & $8,798,565,181$ \\
\hline
\end{tabular}

\subsubsection{The Factors that Affect the Performance of PHES}

In this part, the factors which may have great influence on the performance of PHES will be discussed in detail, with default gap set to $0.05 \%$. An index called output degree is proposed for evaluating.

$$
I_{\text {output }}=\frac{1}{N u m} \sum_{i=1}^{N u m} \text { ang }- \text { output }_{i}^{2}
$$

where avg is the average value of the outputs in one mode. output ${ }_{i}$ represents the output of PHES, which could be positive or negative value depends on its mode. Num is the number of outputs in one mode. The definition of them are:

$$
\begin{gathered}
\text { avg }=\frac{1}{\text { Num }} \sum_{i=1}^{\text {Num }} \text { output }_{i} \\
\text { output }_{t}=\left\{\begin{array}{r}
\text { generating_power } \\
\text { fumping_power generating mode }
\end{array}\right. \\
N u m=\left\{\begin{array}{l}
\text { the total of positive number, for generating mode } \\
\text { the total of negative number, for pumping mode }
\end{array}\right.
\end{gathered}
$$

To describe the variation tendency of PHES, Figures 3 and 4 are taken to describe the data of output degree. The simulation results are exhibited in Figures 3 and 4.

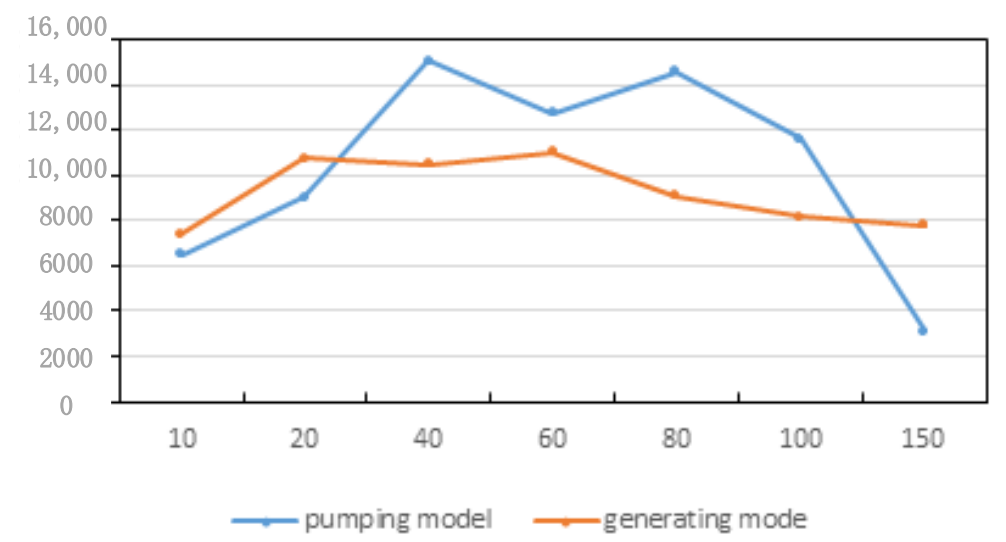

Figure 3. Comparison of PHES in different scale of systems.

From Figure 1, it is obvious that the fluctuation of pumping power is great. After 80-unit case, the blue line decreases dramatically. In Figure 2, the fluctuation of generating power is fierce and it is becoming smooth from $450 \mathrm{MW}$. The results of these figures demonstrate that the output of PHES 
cannot keep increasing without any limitation. These results describes that capacity of PHES and the scale of power systems are the main factors that have a great influence on the performance of PHES. Therefore, a suitable capacity and scale can balance the fluctuation caused by RES better, while it can avoid unnecessary waste of resources at most.

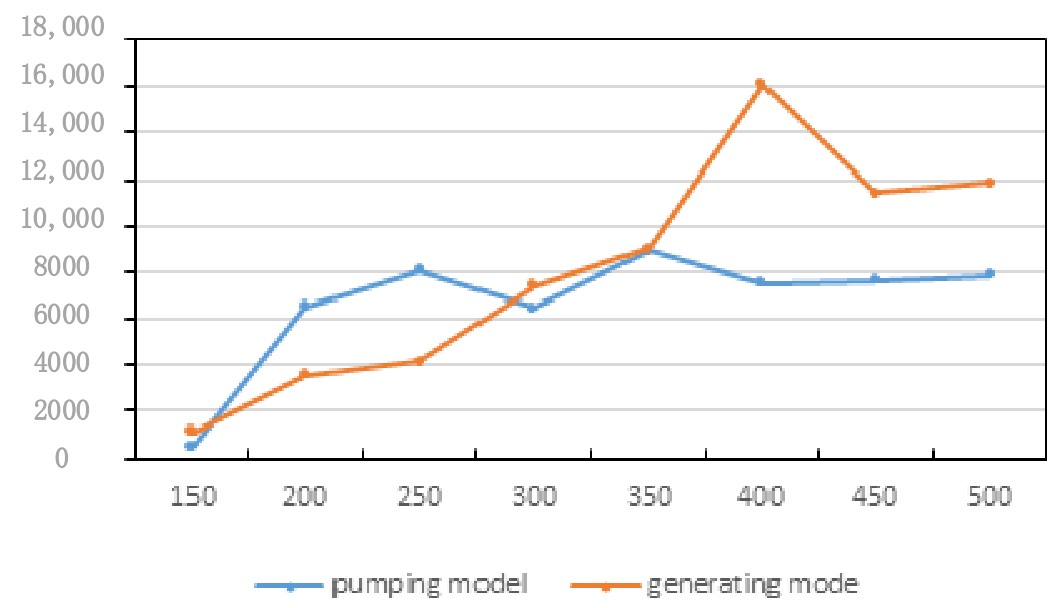

Figure 4. Comparison of PHES output with different capacities.

\section{Conclusions}

Symmetric problem appears in MILP formula if this method is applied to solve the UC model of a power system in which there are identical generators existing. In this paper, an improved MILP approach has been proposed for efficiency promotion by solving the symmetric problem with the method of reforming the hierarchical constraints. To validate the effectiveness of the proposed approach, it has been applied and compared to traditional methods in solving UC problem of power systems with different scales. Then, this approach was applied to investigate the UC problem of a hybrid power system containing thermal, hydro and wind power. The influence of RES integration, as well as impact of PHES has been studied.

Based on experimental results, the following conclusions could be drawn.

(1) The proposed approach was suitable for the large-scale systems of UC as it can obtain a better objective value with less time cost in most cases. To compare with other algorithms, the proposed approach can still maintain its advantages on objective values and time cost.

(2) The integration of RES can decrease the thermal outputs, and furthermore, decrease the operational cost.

(3) As one of the most effective ESSs, PHES can reduce the load fluctuation which caused by RES integration, as well as ensure the stable and reliable operation of the whole system. The performance of PHES is closely related to two factors: The scale of system and the capacity of it. A suitable PHES can avoid unnecessary waste of resources in the real world.

(4) Considering that the change of water-head is ignored in PHES for implementing an ideal model, more studies on UC problem with realistic PHES features would be interesting in future research.

Author Contributions: C.L. provided the funding, idea and led the research work. C.L. and B.F. designed experiments; C.O. carried out experiments; C.O., J.W. and E.G. analyzed experimental results. B.F. and C.O. wrote the manuscript.

Funding: This research was funded by [National Key Research and Development Program of China] grant number [2016YFC0401910] and [National Natural Science Foundation of China] grant number [51679095, 51879111].

Conflicts of Interest: The authors declare no conflicts of interest. 


\section{Nomenclature}

$\begin{array}{ll}T & \text { Scheduling horizon } \\ G e n & \text { Number of generation units } \\ C p_{i, t} & \text { Generating cost (\$) } \\ a_{i}, b_{i}, c_{i} & \text { Parameters of generating } \\ \text { onoff } f_{i, t} & \text { Binary state variable. 1 meaning on and 0 off } \\ p_{i} & \text { Thermal power variable (MW) } \\ C u_{i, t} & \text { Startup cost (\$) } \\ z_{i, k, 1}, z_{i, k, 2} & \text { Hot/cold state variable } \\ H S T_{i}, C S T_{i} & \text { Hot/cold startup cost (\$) } \\ L o a d_{t} & \text { Hourly system demand (MW) } \\ P h_{t} & \text { Hydropower variable (MW) } \\ P w_{t} & \text { Wind power variable (MW) } \\ P_{i, \max }, P_{i, \min } & \text { Maximum/Minimum output of thermal units (MW) } \\ r e s e r v e_{t} & \text { Spinning reserve (MW) } \\ y_{i, t, 1}, y_{i, t, 2} & \text { Startup/Shutdown state variable } \\ D T & \text { Minimum down time (h) } \\ T c o l d & \text { Minimum cold time (h) } \\ V_{t}^{u}, V_{t}^{d} & \text { Up/down reservoir capacity } \\ V_{\min }^{u}, V_{\max }^{u} & \text { Minimum/Maximum capacity of up reservoir } \\ V_{\min }^{d}, V_{\max }^{d} & \text { Minimum/Maximum capacity of down reservoir } \\ u_{1 t}, u_{2 t} & \text { Pumping/Generating mode state variable } \\ \eta_{1}, \eta_{2} & \text { Efficiency of generating/pumping } \\ P h_{t \min }, P h_{t \max } & \text { Minimum/Maximum output of pumped-storage } \\ P P_{\max }, P G_{\max } & \text { plant (MW) } \\ p_{r} & \text { Maximum pumping/generating power (MW) } \\ v_{t} & \text { Rate power of wind (MW) } \\ v_{i}, v_{r} & \text { Cut-in/Cut/off variable (m/sec) } \\ & \end{array}$

\section{References}

1. Alemany, J.; Magnago, F.; Moitre, D.; Pinto, H. Symmetry issues in mixed integer programming based Unit Commitment. Int. J. Electr. Power Energy Syst. 2014, 54, 86-90. [CrossRef]

2. Margot, F. Symmetry in Integer Linear Programming. In Years of Integer Programming; Springer: Berlin/Heidelberg, Germany, 2010; pp. 647-686.

3. Margot, F. Exploiting orbits in symmetric ILP. Math. Program. 2003, 98, 3-21. [CrossRef]

4. Ostrowski, J.; Linderoth, J.; Rossi, F.; Smriglio, S. Orbital branching. Math. Program. 2011, 126, $147-178$. [CrossRef]

5. Yokoyama, R.; Shinano, Y.; Taniguchi, S.; Wakui, T. Search for K-best solutions in optimal design of energy supply systems by an extended MILP hierarchical branch and bound method. Energy 2018. [CrossRef]

6. Lima, R.M.; Novais, A.Q. Symmetry breaking in MILP formulations for Unit Commitment problems. Comput. Chem. Eng. 2016, 85, 162-176. [CrossRef]

7. Ostrowski, J.; Anjos, M.F.; Vannelli, A. Tight Mixed Integer Linear Programming Formulations for the Unit Commitment Problem. IEEE Trans. Power Syst. 2012, 27, 39-46. [CrossRef]

8. Morales-España, G.; Latorre, J.M.; Ramos, A. Tight and compact MILP formulation for the thermal unit commitment problem. IEEE Trans. Power Syst. 2013, 28, 4879-4908. [CrossRef]

9. Lai, X.; Li, C.; Zhang, N.; Zhou, J. A multi-objective artificial sheep algorithm. Neural Comput. Appl. 2018. [CrossRef]

10. Li, C.; Wang, W.; Chen, D. Multi-objective complementary scheduling of Hydro-Thermal-RE power system via a multi-objective hybrid grey wolf optimizer. Energy 2019, 171, 241-255. [CrossRef]

11. Xu, Y.; Li, C.; Wang, Z.; Zhang, N.; Peng, B. Load frequency control of a novel renewable energy integrated micro-grid containing pumped hydropower energy storage. IEEE Access 2018, 6, 29067-29077. [CrossRef] 
12. Reihani, E.; Sepasi, S.; Roose, L.R.; Matsuura, M. Energy management at the distribution grid using a Battery Energy Storage System (BESS). Int. J. Elect. Power Energy Syst. 2016, 77, 337-344. [CrossRef]

13. Luo, X.; Wang, J.; Dooner, M.; Clarke, J. Overview of current development in electrical energy storage technologies and the application potential in power system operation. Appl. Energy 2015, 137, 511-536. [CrossRef]

14. Wang, W.; Li, C.; Liao, X.; Qin, H. Study on unit commitment problem considering pumped storage and renewable energy via a novel binary artificial sheep algorithm. Appl. Energy 2017, 187, 612-626. [CrossRef]

15. Frangioni, A.; Gentile, C.; Lacalandra, F. Tighter approximated MILP formulations for unit commitment problems. IEEE Trans. Power Syst. 2009, 24, 105-113. [CrossRef]

16. Frangioni, A.; Gentile, C. Perspective cuts for a class of convex 0-1 mixed integer programs. Math Program. Ser. A 2006, 106, 225-236. [CrossRef]

17. Wang, Z.; Li, C.; Lai, X.; Zhang, N.; Xu, Y.; Hou, J. An integrated start-up method for pumped storage units based on a novel artificial sheep algorithm. Energies 2018, 11, 151. [CrossRef]

18. Sheng, S.; Sun, X. An economic dispatching strategy of peak load shifting by wind farm and pumped storage plant. Power Syst. Technol. 2014, 38, 2484-2489.

19. Ma, T.; Yang, H.; Lu, L.; Peng, J. Technical feasibility study on a standalone hybrid solar-wind system with pumped hydro storage for a remote island in Hong Kong. Renew. Energy 2014, 69, 7-15. [CrossRef]

20. Sherali, H.D.; Smith, J.C. Improving Discrete Model Representations via Symmetry Considerations. Informs 2001, 47, 1396-1407. [CrossRef]

21. Senjyu, T.; Shimabukuro, K.; Uezato, K.; Funabashi, T. A fast technique for unit commitment problem by extended priority list. IEEE Trans. Power Syst. 2003, 18, 882-888. [CrossRef]

22. Ongsakul, W.; Petcharaks, N. Unit commitment by enhanced adaptive Lagrangian relaxation. IEEE Trans. Power Syst. 2004, 19, 620-628. [CrossRef]

23. Seki, T.; Yamashita, N.; Kawamoto, K. New Local Search Methods for Improving the Lagrangian-RelaxationBased Unit Commitment Solution. IEEE Trans. Power Syst. 2010, 25, 272-283. [CrossRef]

24. Yang, L.; Jian, J.; Zheng, H.; Han, D. A Sub Hyper-cube Tight Mixed Integer Programming Extended Cutting Plane Method for Unit Commitment. Proc. CSEE 2013, 33, 99-108. 